DIE VERWALTUNG 50 (2017), 51-76

Duncker \& Humblot, 12165 Berlin

\title{
DER KODIFIKATIONSENTWURF FÜR EIN EU-VERWALTUNGSVERFAHRENSRECHT AUS SCHWEIZERISCHER PERSPEKTIVE
}

\author{
Von Benjamin Schindler, St. Gallen ${ }^{1}$
}

\section{Vorbemerkungen aus schweizerischer Sicht}

Die Schweiz ist nicht Mitglied der Europäischen Union und dürfte dies in absehbarer Zukunft auch nicht werden. Es erstaunt daher nicht, dass der ReNEUAL-Musterentwurf für ein EU-Verwaltungsverfahrensrecht ${ }^{2}$ in der Schweiz bislang kaum Beachtung fand. Wenig überraschend ist auch, dass die schweizerische Rechtsordnung im Rahmen der Ausarbeitung des Musterentwurfs nicht berücksichtigt wurde. Dieses auf Gegenseitigkeit beruhende Desinteresse ist bedauerlich und lässt sich sachlich kaum rechtfertigen. Einerseits könnte der Musterentwurf für die schweizerische Rechtsordnung sehr wohl relevant werden. Und andererseits ließe sich die europäische Diskussion durchaus um einige helvetische Aspekte bereichern.

\section{Relevanz des Musterentwurfs für die schweizerische Rechtsordnung}

Die Europäische Union und die Schweiz sind geographisch, gesellschaftlich, wirtschaftlich und kulturell eng miteinander verbunden. Diese Bindungen fanden seit 1972 ihren rechtlichen Niederschlag in zahlreichen , bilateralen“3 Verträgen. Dieser ,,bilaterale Weg“ wurde von der Schweiz seit 1992 intensiviert verfolgt, nachdem Volk und Stände einen Beitritt der Schweiz zum

1 Erweiterte Fassung des Vortrags im „Gesprächskreis Verwaltung“ der Vereinigung der Deutschen Staatsrechtslehrer am 5. Oktober 2016 in Linz. Ein besonderer Dank gilt meinem Mitarbeiter Dr. Tobias Tschumi für die unverzichtbare Unterstützung bei der Vorbereitung sowie meinem Kollegen Prof. Dr. Matthias Oesch für die wertvollen Hinweise aus europarechtlicher Sicht.

2 Schneider/Hofmann/Ziller (Hrsg.), ReNEUAL - Musterentwurf für ein EU-Verwaltungsverfahrensrecht, Research Network on EU Administrative Law (ReNEUAL), 2015.

3 Der gemeinhin verwendete Begriff ,,bilateral“ erweckt den Eindruck von lediglich zwei Vertragsparteien (Schweiz und EU). Dies ist indes nicht für alle Verträge zutreffend. Bei einzelnen Abkommen (etwa Personenfreizügigkeit) sind auch die Mitgliedstaaten der EU Vertragsparteien (sog. ,gemischte Abkommen“). Vgl. Jaag/Hänni, Europarecht: Die europäischen Institutionen aus schweizerischer Sicht, 4. Aufl. 2015, Rn. 4008. 
Europäischen Wirtschaftsraum (EWR) abgelehnt hatten ${ }^{4}$. Die Staatsverträge betreffen inzwischen zahlreiche Politikfelder: Freihandel (1972), Versicherungswesen (1989), Personenfreizügigkeit, Technische Handelshemmnisse, Öffentliches Beschaffungswesen, Landwirtschaft, Landverkehr, Luftverkehr, Forschung (sog. „Bilaterale I“, in Kraft seit 2002), Schengen/Dublin, Zinsbesteuerung, Betrugsbekämpfung, Umwelt, Statistik, Filmförderung

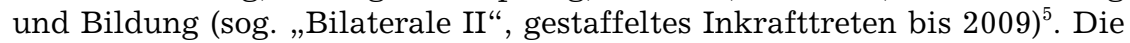
Verträge zielen in erster Linie auf eine Liberalisierung der grenzüberschreitenden Märkte, die Behördenkooperation sowie die Rechtsharmonisierung. Sie führen dazu, dass die Schweiz in großem Umfang unionsrechtliche Regelungen übernimmt, nicht jedoch umgekehrt ${ }^{6}$. In gewisser Hinsicht erscheint die Schweiz somit als „Zugewandter Ort“ der Europäischen Union”. Bislang wenige Auswirkungen haben die Vertragswerke indes auf das Verfahrensrecht und den Rechtsschutz. Durchführung und Überwachung der Verträge basieren weitgehend auf traditionellen völkerrechtlichen Grundsätzen ${ }^{8}$. Anwendung und Vollzug der Abkommen liegen auf dem jeweiligen Hoheitsgebiet bei den nationalen Behörden bzw. den Behörden der Union. Verfahren und Rechtsschutz richten sich daher nach nationalem Verfahrens- und Gerichtsorganisationsrecht. Entscheide schweizerischer Gerichte können nicht an den EuGH weitergezogen werden und die Gerichte haben auch keine Möglichkeit, ein Vorabentscheidungsverfahren vor dem EuGH einzuleiten ${ }^{9}$. Während der Individualrechtsschutz über die nationalen Gerichte sicherzustellen ist, sollen Streitigkeiten zwischen den Vertragsparteien grundsätzlich in Gemischten Ausschüssen beigelegt werden; die Möglichkeit einer Vertragsverletzungsklage vor einem Gericht besteht nicht ${ }^{10}$. Die bisherigen „bilateralen“

4 Vgl. Oesch, Europarecht, Bd. I: Grundlagen, Institutionen, Verhältnis SchweizEU, 2015, Rn. 892.

5 Das Eidgenössische Departement für auswärtige Angelegenheiten veröffentlicht regelmäßig eine kommentierte Übersicht über die bilateralen Abkommen zwischen der Schweiz und der EU (www.eda.admin.ch/dea/de/home/bilaterale-abkommen.html). Zur teilweise noch ausstehenden Ratifikation von Seiten einzelner Mitgliedstaaten der EU vgl. Oesch, Europarecht (FN 4), Rn. 901.

6 Jaag/Hänni, Europarecht (FN 3), Rn. 4013 f.; Oesch, Europarecht (FN 4), Rn. 893.

7 Vgl. Cottier/Liechti, in: Breuss/Cottier/Müller-Graff (Hrsg.), Die Schweiz im europäischen Integrationsprozess, 2008, S. 39 (53). Als „Zugewandte Orte“ wurden in der Alten Eidgenossenschaft diejenigen Bündnispartner bezeichnet, welche mit der Eidgenossenschaft vertraglich zwar eng verbunden waren, aber nicht als vollwertige Mitglieder galten und im institutionellen Forum der ,Tagsatzung“ über kein Stimmrecht verfügten. Dieser vertragliche Sonderstatuts geriet mit der Ausbildung moderner Staatlichkeit unter Druck und mündete entweder in einer „Vollmitgliedschaft" als schweizerischer Kanton (so Genf) oder einer Entkoppelung (so beim heute französischen Mülhausen oder dem heute deutschen Rottweil). Vgl. Würgler, Die Tagsatzung der Eidgenossen: Politik, Kommunikation und Symbolik einer repräsentativen Institution im europäischen Kontext (1470-1798), 2013, S. 108 ff.

8 Vgl. Oesch, Europarecht (FN 4), Rn. 905.

9 Jaag/Hänni, Europarecht (FN 3), Rn. 4022 ff., 4046 ff.

$10 \mathrm{Jaag} / H a ̈ n n i$, Europarecht (FN 3), Rn. 4025 ff., 4049 f.; Oesch, Europarecht (FN 4), Rn. 907. 
Verträge vermögen gerade mit Blick auf den Rechtsschutz und die Institutionen der Streitbeilegung nicht zu befriedigen. Zwischen der starken materiellrechtlichen Teilintegration der Schweiz in die EU und dem weitgehend fehlenden institutionellen und verfahrensrechtlichen Rahmen besteht eine offenkundige Diskrepanz. Ein „Rahmenabkommen“, welches sich diesen institutionellen Fragen für alle Politikbereiche in grundlegender Art und Weise annimmt, erscheint daher aus Sicht der Schweiz wie der EU dringend gebo$\operatorname{ten}^{11}$.

Eine Ausnahme zum oben Dargestellten besteht beim Luftverkehrsabkommen (LVA). Hier verfügen die Organe der EU auch gegenüber der Schweiz über gewisse Befugnisse im Bereiche des Vollzugs ${ }^{12}$. Dort finden die entsprechenden Verfahrensvorschriften des Europarechts bereits heute Anwendung. Hier könnte also auch der ReNEUAL-Musterentwurf - soweit nicht die bereits heute geltenden bereichsspezifischen Verfahrensvorschriften vorgehen - künftig seine Wirkung entfalten. Punktuell kann der Musterentwurf aber auch im Rahmen der Umsetzung der übrigen „bilateralen Verträge“ auf die schweizerische Rechtsordnung einwirken. So sieht etwa die schweizerische Verordnung zum Schengener Informationssystem vor, dass der Eidgenössische Datenschutz- und Öffentlichkeitsbeauftragte (EDÖB) eng mit dem Europäischen Datenschutzbeauftragten zusammenarbeitet ${ }^{13}$. Diese Zusammenarbeit könnte sich künftig am Maßstab von Art. VI-38 des Musterentwurfs orientieren.

Neben denjenigen Politikfeldern, in welchen die Schweiz vertraglich mit der EU verbunden ist, orientiert sich die Schweizer Rechtsetzung auch in zahlreichen anderen Bereichen inhaltlich am europäischen Recht (sog. ,autonomer Nachvollzug“ von EU-Recht). Vor allem aus wirtschaftlichen Überlegungen soll ein Regulierungsgefälle zwischen der Schweiz und der EU vermieden werden. Im diesem Sinne wurden z. B. diejenigen Änderungen der schweizerischen Rechtsordnung, welche bei einem Beitritt zum EWR hätten vorgenommen werden müssen, auf freiwilliger Basis vollzogen (sog. „Swisslex“-Vorlage). Der Bundesrat (Bundesregierung) ist zudem verpflichtet, bei jeder Gesetzesvorlage ans Parlament zu erläutern, in welchem „Verhältnis zum europäischen Recht" diese steht ${ }^{14}$. Praktisch in sämtlichen Bereichen

11 Vgl. Jaag/Hänni, Europarecht (FN 3), Rn. 4052; Oesch, Europarecht (FN 4), Rn. 956 ff. Ein entsprechendes Rahmenabkommen wird von der Schweizerischen Volkspartei (SVP) bekämpft. Die Chancen eines Abkommens scheinen seit der Annahme der von der SVP lancierten Volksinitiative „Gegen Masseneinwanderung“ vom 9. Februar 2014 deutlich geschwunden.

12 Art. 11 Abs. 1 und 18 Abs. 2 des Abkommens zwischen der Schweizerischen Eidgenossenschaft und der Europäischen Gemeinschaft über den Luftverkehr vom 21. Juni 1999, SR (= Systematische Rechtssammlung des Bundes) 0.748.127.192.68; Oesch, Europarecht (FN 4), Rn. 231, 908.

13 Art. 55 Abs. 3 der Verordnung über den nationalen Teil des Schengener Informationssystems (N-SIS) und das SIRENE-Büro (N-SIS-Verordnung) vom 8. März 2013 (SR 362.0).

14 Art. 141 Abs. 2 Bst. a des Bundesgesetzes über die Bundesversammlung vom 13. Dezember 2002 (Parlamentsgesetz; SR 171.10). 
der Gesetzgebung des Bundes ist daher der Einfluss europäischen Rechts sehr deutlich spürbar. Davon erfasst ist nicht nur das materielle Recht, sondern auch das Verfahrensrecht ${ }^{15}$. Auch wenn es sich streng genommen beim nachvollzogenen EU-Recht um rein schweizerisches Recht handelt, bewirkt der Nachvollzug bereichsspezifisch eine parallele Rechtslage.

\section{Relevanz der schweizerischen Perspektive für die Arbeit am Musterentwurf}

Der breit angelegte Rechtsvergleich, welcher Grundlage des Musterentwurfs ist, kam ohne das Schweizer Recht aus ${ }^{16}$. Das macht auf den ersten Blick durchaus Sinn, ist die Schweiz doch kein Vollmitglied der EU. Bei genauerem Hinsehen zeigen sich aber in vielfacher Hinsicht Ähnlichkeiten zwischen der Schweiz und der EU - ja in gewisser Hinsicht könnte man die Schweiz auch als „Europe en miniature“ beschreiben. Ein schweizerischer Seitenblick auf den Musterentwurf könnte daher auch für die Europäische Union lohnenswert erscheinen ${ }^{17}$.

Die Schweiz hat eine ausgeprägt föderalistische Struktur. Die Kantone der Schweiz sind nicht die Folge einer nachträglichen Dezentralisierung. Vielmehr reichen ihre Wurzeln als eigene Staatswesen weit in die Geschichte zurück und der heutige Bundesstaat ist über Jahrhunderte aus einem komplexen und heterogenen Bündnissystem herausgewachsen. So ist die ausgesprochene Eigenstaatlichkeit der Kantone bis heute ein prägendes Merkmal des schweizerischen Föderalismus. Die Schweiz kann als „le pays le plus décentralisé d'Europe"18 bezeichnet werden. Auf verfassungsrechtlicher Ebene kommt dies auch in Art. 3 der Bundesverfassung (BV) zum Ausdruck, welcher die Tocquevillesche Vorstellung von ,geteilter Souveränität“ zum Ausdruck bringt ${ }^{19}$. Die föderalistische Struktur zeigt sich auch darin, dass Verwaltungstätigkeit in der Schweiz - ähnlich der $\mathrm{EU}^{20}$ - eine komplexe Verbundaufgabe ist. Die Umsetzung von Bundesrecht erfolgt seit 1874 vornehmlich

15 So z.B. Art. 20 des Bundesgesetzes über die technischen Handelshemmnisse (THG) vom 6. Oktober 1995 (SR 946.51). Er regelt die Marktüberwachung von nach ausländischen technischen Vorschriften hergestellten Produkten und orientiert sich an der entsprechenden EG-Verordnung Nr. 764/2008. Vgl. Botschaft (des Bundesrats) zur Teilrevision des Bundesgesetzes über die technischen Handelshemmnisse vom 25. Juni 2008, BBl (= Bundesblatt) 2008 7275, 7304.

16 Vgl. Schneider/Hofmann/Ziller (Hrsg.), ReNEUAL - Musterentwurf (FN 2), S. 8 Rn. 28 ff., S. 17 Rn. 62 f.

17 Vgl. Blondel, in: Kübler/Stojanović (Hrsg.), Democracy in the European Union A Contribution from the Swiss Perspective, 2014, S. $69 \mathrm{ff}$.

18 Fromont, Droit administratif des états européens, 2006, S. 43.

19 Art. 3 der Bundesverfassung vom 18. April 1999 (SR 101), welcher praktisch wortgleich Art. 3 der ersten Bundesverfassung von 1848 entspricht, lautet: „Die Kantone sind souverän, soweit ihre Souveränität nicht durch die Bundesverfassung beschränkt ist; sie üben alle Rechte aus, die nicht dem Bund übertragen sind."

20 Vgl. Schneider/Hofmann/Ziller (Hrsg.), ReNEUAL - Musterentwurf (FN 2), S. 8 Rn. $26 \mathrm{f}$. 
durch die Kantone und Gemeinden im Rahmen des Vollzugs- oder Umsetzungsföderalismus ${ }^{21}$. Der Bund selber verfügt nur in wenigen Bereichen über eigene Vollzugsorgane (etwa Zoll oder Asylwesen). In zahlreichen Politikfeldern, welche bundesrechtlich nicht vereinheitlicht wurden (etwa Bildung oder Polizeiwesen), kooperieren die Kantone zudem auf freiwilliger Basis. Die Schweiz kann somit als Prototyp eines sog. „Verwaltungsverbunds“ bezeichnet werden ${ }^{22}$. Die Schweiz ist schließlich von Staaten der Europäischen Union umgeben und Deutschland und Frankreich haben maßgeblichen Einfluss auf die Entwicklung des schweizerischen Verwaltungsrechts ausgeübt - in geringerem Ausmaß auch Österreich und Italien ${ }^{23}$. Schließlich hat die EMRK seit ihrem Inkrafttreten für die Schweiz (1974) die Anforderungen an den Rechtsschutz im Verwaltungsrecht maßgeblich mitgeprägt ${ }^{24}$. Gerade in verwaltungsrechtlicher und verwaltungsprozessualer Hinsicht ist die Schweiz daher ein europäischer "Schmelztiegel“.

Schließlich hat die Schweiz eine bald 170-jährige Tradition im Formulieren mehrsprachiger Gesetzestexte, welche zugleich den Besonderheiten eines vielgestaltigen Föderalismus und unterschiedlichen Verwaltungskulturen Rechnung tragen müssen. Dazu kommt - und dies dürfte eine Schweizer Eigenheit sein - die stark direktdemokratisch geprägte Ausgestaltung des Gesetzgebungsverfahrens. Gesetzesvorlagen sind im Bund, in den Kantonen und in den Gemeinden vielfach Gegenstand von Volksabstimmungen. Die Mehrsprachigkeit und das Bedürfnis nach Verständlichkeit haben in der Schweiz eine besondere Sensibilität für die Gesetzessprache bewirkt. Das Leitmotiv der Gesetzessprache ist: „Denken wie ein Philosoph und Schreiben wie ein Bauer “25. Vorbild guter Gesetzgebung ist das von Eugen Huber (18491923) entworfene Schweizerische Zivilgesetzbuch von $1907\left(\mathrm{ZGB}^{26}\right)$; es reflektiert sozusagen die „bonnes pratiques“ (bzw. „,best practice“) der Rechtsetzung. Auch das Verwaltungsverfahrensgesetz des Bundes $\left(\mathrm{VwVG}^{27}\right)$ ist in dieser Tradition entstanden. Es erscheint daher sinnvoll, das VwVG zum Ausgangspunkt einer spezifisch helvetischen Perspektive zu nehmen.

21 Vgl. Art. 46 Abs. 1 BV: „Die Kantone setzen das Bundesrecht nach Maßgabe von Verfassung und Gesetz um."

22 Schindler, in: Bogdandy/Cassese/Huber (Hrsg.), Handbuch Ius Publicum Europaeum, Bd. III, 2010, § 49 Rn. 28. Zum Verbundmodell Schmidt-Aßmann, in: ders., Aufgaben und Perspektiven verwaltungsrechtlicher Forschung, 2006, S. 466 ff.

23 Schindler, in: Handbuch Ius Publicum Europaeum III (FN 22), § 49 Rn. 19 ff.

24 Art. 6 EMRK führte in erster Linie zu einem Entwicklungsschub für die Verwaltungsgerichtsbarkeit - dieser strahlte aber auch auf das vorgelagerte Verwaltungsverfahren aus. Vgl. Herzog, Art. 6 EMRK und kantonale Verwaltungsrechtspflege, 1995; Schweizer, Zeitschrift für Schweizerisches Recht (ZSR) 1993 II, S. 577 ff.

25 Vgl. die Beiträge in: Lötscher/Nussbaumer (Hrsg.), Denken wie ein Philosoph und schreiben wie ein Bauer: Sprache, mit der ein Staat zu machen ist, 2007; Alain Griffel (Hrsg.), Vom Wert einer guten Gesetzgebung, 2014.

26 Schweizerisches Zivilgesetzbuch vom 10. Dezember 1907 (SR 210).

27 Bundesgesetz über das Verwaltungsverfahren vom 20. Dezember 1968 (SR 172.021). 


\section{Verwaltungsverfahrensgesetz des Bundes (VwVG) von 1968 als Kodifikationsbeispiel}

Das bis heute in seinen Grundzügen weitgehend unverändert geltende Bundesgesetz über das Verwaltungsverfahren stammt aus dem Jahr 1968. Den Anstoß zur Kodifikation lieferte eine Resolution, welche am Schweizerischen Juristentag 1950 verabschiedet wurde ${ }^{28}$, sowie ein Vorentwurf von Max Imboden $(1915-1969)^{29}$. Der Impuls zur Reform kam also - wie beim Musterentwurf für ein EU-Verwaltungsverfahrensrecht - aus Kreisen der Wissenschaft. Damit sich die Politik des Anliegens annahm, brauchte es indes (wie leider so oft) einen Skandal. Diesen lieferte 1964 das Eidgenössische Militärdepartement mit der Beschaffung von neuen Kampfflugzeugen. Regierung und Verwaltung hatten es versäumt, das Parlament über alle Kosten ausreichend und rechtzeitig zu informieren. Der dadurch hervorgerufene Ärger auf Seiten des Parlaments lieferte in der Folge die bislang fehlende Energie zum Ausbau der Verwaltungsgerichtsbarkeit und zur Kodifikation des Verwaltungsverfahrens ${ }^{30}$.

Aufgrund dieser Vorgeschichte zielte die Verfahrenskodifikation zum einen darauf ab, das Vertrauen in die Verwaltung wieder herzustellen. Andererseits bezweckte das neue Gesetz auch eine Vereinheitlichung. Zwar bestanden bereits zahlreiche Verfahrensvorschriften im Bundesrecht, doch waren diese durch „Zersplitterung und Lückenhaftigkeit“ gekennzeichnet ${ }^{31}$. Hier besteht eine offensichtliche Parallele zum Kodifikationsvorhaben in der EU: Auch die Regeln des EU-Verfahrensrechts sind durch „signifikante Fragmentierung“ und „Regelungslücken“ gekennzeichnet ${ }^{32}$. Die mit dem Erlass des VwVG im Jahr 1968 angestrebte Vereinheitlichung sollte aber nicht „auf der grünen Wiese“ erfolgen; vielmehr ließ sich der Gesetzgeber von den bestehenden Regeln im Bund, in den Kantonen und von der Praxis des Bundesgerichts inspirieren. Ähnlich wie der österreichische Verwaltungsgerichtshof hatte das Bundesgericht seit 1874 in einer kreativen Rechtsprechung aus dem allgemeinen Rechtsgleichheitsgebot der Bundesverfassung zahlreiche verfahrensrechtliche Minimalgarantien abgeleitet ${ }^{33}$. Das Rechts-

28 Resolution des Schweizerischen Juristenvereins vom 11. September 1950, ZSR 1950 II, S. 442a f.

${ }_{29}$ Zur Vorgeschichte und den verschiedenen Entwürfen Imbodens: Schindler, in: Auer/Müller/ders. (Hrsg.), Kommentar zum Bundesgesetz über das Verwaltungsverfahren, 2008, Einleitung Rn. 6.

30 Bericht der vom Nationalrat und vom Ständerat eingesetzten Kommissionen an die Eidgenössischen Räte über die Abklärung der Mirage-Angelegenheit vom 1. September 1964, BBl 1964 II 273 (344 f., 348).

31 Botschaft des Bundesrates an die Bundesversammlung über das Verwaltungsverfahren vom 24. September 1965, BBl 1965 II 1348 (1352).

32 Vgl. Schneider/Hofmann/Ziller (Hrsg.), ReNEUAL - Musterentwurf (FN 2), S. 4 Rn. 14 .

33 Georg Müller, in: Aubert/Eichenberger/Müller/Rhinow/Schindler (Hrsg.), Kommentar zur Bundesverfassung der Schweizerischen Eidgenossenschaft vom 
gleichheitsgebot übernahm damit eine ähnliche Funktion wie Art. 41 der Grundrechtecharta in der Union ${ }^{34}$. Auch der Musterentwurf verfolgt ja das Anliegen, die bereits bestehenden und vom EuGH entwickelten allgemeinen Grundsätze des EU-Verfahrensrechts zu kodifizieren ${ }^{35}$. Eine weitere Parallele zum Musterentwurf besteht schließlich im Geltungsbereich des VwVG dieses beschränkt sich auf das Verfahren der Bundesbehörden. Für Behörden von Kantonen und Gemeinden gilt das VwVG grundsätzlich nicht; auch dann nicht, wenn diese materielles Bundesrecht anwenden ${ }^{36}$. Das VwVG zielt damit nicht auf eine Rechtsvereinheitlichung im föderalistischen Mehrebenensystem. Auch hier entspricht das VwVG dem Musterentwurf, welcher sich auf die Organe, Einrichtungen, Ämter und Agenturen der EU beschränkt - zumindest soweit das Verfahren der Einzelfallentscheidung (Buch III) geregelt werden soll. Allerdings hatte das VwVG auf das Verfahrensrecht der Kantone einen starken ,spill over-Effekt“37. Ein solcher wäre voraussichtlich auch bei einer Kodifikation des EU-Verwaltungsverfahrens auf die mitgliedstaatlichen Rechtsordnungen zu erwarten und ist in Art. I-3 des Musterentwurfs bereits angelegt ${ }^{38}$.

Die Europäische Bürgerbeauftragte Emily O'Reilly hat in ihrem Vorwort zum Musterentwurf für ein EU-Verwaltungsverfahrensrecht festgestellt, dass sich die Unionsbürgerschaft in einer „Krise“ befinde. Als wesentlichen Aspekt dieser Krise bezeichnet sie den Umstand, „dass vielen Bürgerinnen und Bürgern die EU und ihre Institutionen als fremd und fern erscheinen " 39 . Die Erfahrungen in der Schweiz zeigen, dass die Kodifikation des Verwaltungsverfahrens und damit die Überführung von Verwaltungsabläufen und der Kommunikation zwischen Behörden und Individuen in einheitliche, stabile und transparente Bahnen, eine mögliche Reaktion auf ein gestörtes Verhältnis zwischen Individuum und Gemeinwesen sein kann. Das schweizerische Beispiel macht zudem deutlich, dass „Bürgerfreundlichkeit“" ${ }^{40}$ auch darin zum Ausdruck kommt, wie eine Kodifikation ausgestaltet ist und in welche Sprache sie gefasst wird. Die folgenden Ausführungen versuchen, einzelne

29. Mai 1874, 1995, Art. 5 Rn. 85 ff.; Schindler, in: Kommentar zum Bundesgesetz über das Verwaltungsverfahren (FN 29), Einleitung Rn. 3.

$34 \mathrm{Zu}$ den Parallelen: Thurnherr, Schweizerisches Jahrbuch für Europarecht 2011/ 2012, S. 447 (462).

35 Vgl. Schneider/Hofmann/Ziller (Hrsg.), ReNEUAL - Musterentwurf (FN 2), S. 7 Rn. 23.

36 Zum Grundsatz: Art. 1 Abs. 1 und 2 VwVG. In (seltenen) Ausnahmefällen finden einzelne Bestimmungen des VwVG auch auf kantonale Behörden Anwendung: Art. 1 Abs. 3 VwVG.

37 Schindler, in: Kommentar zum Bundesgesetz über das Verwaltungsverfahren (FN 29), Einleitung Rn. 15; Thurnherr, Basler Juristische Mitteilungen (BJM), 2013, S. 217 (224 f.).

38 Vgl. Pünder, in: Ehlers/ders. (Hrsg.), Allgemeines Verwaltungsrecht, 15. Aufl. 2016, S. 405 (433 Rn. 30).

39 O'Reilly, in: ReNEUAL - Musterentwurf (FN 2), S. IX.

40 Der Begriff ist insofern nicht ganz zutreffend, als das Verwaltungsverfahren auch das Verhältnis zu Personen regelt, welche nicht Bürgerinnen oder Bürger sind. 
Aspekte des EU-Kodifikationsentwurfs vor diesem schweizerischen Erfahrungshorizont kritisch zu beleuchten.

\section{Regelungskonzept: Nachführung oder Innovation?}

\section{Verfahrensrecht als stabilisierendes Normgerüst}

Die Verfasser des ReNEUAL-Musterentwurfs erheben den Anspruch, eine „innovative Kodifikation“ vorzuschlagen. Der Entwurf beschränkt sich also bewusst nicht auf die „Konsolidierung“ (,codification à droit constant“) der bereits bestehenden Normen und der Rechtsprechung ${ }^{41}$. Hier unterscheidet sich der Entwurf auf den ersten Blick grundlegend vom VwVG. Mit dem VwVG wurde das Ziel einer „Minimalkodifikation“42 angestrebt, welche das bereits geltende Recht konsolidieren und vereinheitlichen sollte. Der Urheber des Vorentwurfs (Max Imboden) bezeichnete dieses Anliegen vor der Vereinigung der Deutschen Staatsrechtslehrer selber als „,bescheidenes gesetzgeberisches Programm“43. Er rechtfertigte dies mit dem weiten Anwendungsbereich des Gesetzes, welches praktisch sämtliche Sachbereiche des Besonderen Verwaltungsrechts erfasse ${ }^{44}$. Auch außerhalb des Verwaltungsverfahrens hat dieses Konzept der „Nachführung“ in der Schweiz eine gewisse Tradition und lag etwa der Totalrevision der Bundesverfassung von $1999 \mathrm{zu}$ Grunde $^{45}$. Aus einer helvetisch-pragmatischen Sicht sprechen mit Blick auf die Kodifikation des EU-Verwaltungsverfahrens einige Argumente für eine bloße Nachführung:

Das Verwaltungsverfahrensrecht ist im deutschsprachigen Raum nicht durch besondere Innovation und Dynamik geprägt. Diese Feststellung gilt gerade mit Blick auf das schweizerische VwVG, das österreichische Allgemeine Verwaltungsverfahrensgesetz $\left(\mathrm{AVG}^{46}\right)$ von 1925 (wiederverlautbart 1991) und das deutsche Verwaltungsverfahrensgesetz (VwVfG) von 1976. Man mag dieses Beharren mit Blick auf die schlummernden und ungenutzten Steuerungsressourcen bedauern. Möglicherweise würde dabei aber eine zentrale Funktion des Verwaltungsverfahrensrechts verkannt. Otto Mayer beginnt seine Ausführungen zum Verwaltungsakt mit einer programmatischen Aussage: „Im Gegensatz zur vorausgehenden Entwicklungsstufe hat unser Rechtsstaat nicht bloß die flutende Masse der Verwaltungstätigkeit einge-

41 Schneider/Hofmann/Ziller (Hrsg.), ReNEUAL - Musterentwurf (FN 2), S. 2 Rn. 4 und S. 5 Rn. 17.

42 Botschaft VwVG (FN 27), S. 1362.

43 Imboden, Diskussionsvotum, VVDStRL 17 (1958), S. 215.

44 Imboden, Diskussionsvotum, VVDStRL 17 (1958), S. 216.

45 Der Begriff „Nachführung“ wird gemeinhin auf Kurt Eichenberger (1922-2005) zurückgeführt (Artikel in der Neuen Zürcher Zeitung vom 12. Mai 1986, S. 19) und war die Antwort auf zahlreiche gescheiterte Versuche, die Bundesverfassung einer gesamthaften Revision (,Totalrevision“) zu unterziehen.

46 Allgemeines Verwaltungsverfahrensgesetz, BGBl. Nr. 51/1991. 
dämmt, sondern er lässt auch noch mitten drin fort und fort feste Punkte auftauchen, welche dem Einzelnen Halt gewähren und ihn darüber sicherstellen, wohin es geht." 47

Ist es nicht gerade eine Kernfunktion des Verwaltungsverfahrens in einem höchst dynamischen und heterogenen Rechtsgebiet wie dem Verwaltungsrecht geordnete und vorhersehbare Bahnen der Kommunikation, Informationsgewinnung und Entscheidungsfindung vorzugeben und zu strukturieren? Ließe sich - in Anlehnung an Otto Mayer - nicht mit guten Gründen behaupten: „Materielles Verwaltungsrecht vergeht, Verwaltungsverfahrensrecht besteht "48? Rückt man diese stabilisierende Funktion des Verfahrensrechts in den Vordergrund, dann sollte das Anliegen eines Musterentwurfs in erster Linie darin bestehen, bewährte Verfahrensregeln in eine Kodifikation zu überführen, zu konsolidieren und transparent zu machen.

\section{Verfahrensrecht als Steuerungsressource}

Dem Verfahrensrecht kommt allerdings nicht nur eine stabilisierende Funktion zu - es kann auch eine Steuerungsressource $\operatorname{sein}^{49}$. Ausgerechnet die älteste deutschsprachige Kodifikation des Verwaltungsverfahrens, das liechtensteinische Gesetz über die allgemeine Landesverwaltungspflege $\left(\mathrm{LVG}^{50}\right)$ aus dem Jahr 1922, ist Ausdruck einer gesetzgeberischen Konzeption, durch welche das Verhältnis zwischen staatlichen Behörden und Individuen bewusst und grundlegend neu gestaltet werden sollte ${ }^{51}$. Die innovative Steuerungskraft des Verfahrensrechts besteht zuerst einmal darin, wie weit der sachliche Anwendungsbereich einer Kodifikation gezogen wird oder nicht ${ }^{52}$. Aus schweizerischer Sicht kann als ,,innovativ“ sicher die umfassende Perspektive gelten, mit welcher der ReNEUAL-Musterentwurf neben der Ein-

47 Mayer, Deutsches Verwaltungsrecht, I. Band, 3. Aufl. 1924, S. 92 f.

48 Mayer, Verwaltungsrecht (FN 47), Vorwort zur dritten Auflage: „Verfassungsrecht vergeht, Verwaltungsrecht besteht".

49 Jochum, Verwaltungsverfahrensrecht und Verwaltungsprozeßrecht, 2004, S. 84. Der Begriff der Steuerungsressource wurde v. a. durch Schmidt-Aßmann mit Blick auf das Verwaltungsorganisationsrecht geprägt: Schmidt-Aßmann, in: ders./HoffmannRiem (Hrsg.), Verwaltungsorganisationsrecht als Steuerungsressource, 1997, S. 9 ff.

50 Gesetz vom 21. April 1922 über die allgemeine Landesverwaltungspflege (die Verwaltungsbehörden und ihre Hilfsorgane, das Verfahren in Verwaltungssachen, das Verwaltungszwangs- und Verwaltungsstrafverfahren), LR (= Liechtensteinische Rechtsvorschriften) 172.020 .

51 Hintergrund des Gesetzes ist die mit der Verfassungsreform von 1921 verbundene Stärkung von Demokratie, Rechtsstaat und Eigenstaatlichkeit, nachdem die bislang prägende Habsburgermonarchie in sich zusammengebrochen war. Zum Entstehungskontext des Gesetzes vgl. Schädler, Arbeitspapiere Liechtenstein-Institut, 54, 2016, S. 19 ff., 27 ff., 33 ff., 43 ff.

52 Dem liechtensteinischen LVG lag seinerzeit der Ansatz einer umfassenden Kodifikation zu Grunde; es umfasst auch Bestimmungen organisatorischer Art, eine eingehende Regelung von Verwaltungszwang und Vollstreckung sowie des Verwaltungsstrafverfahrens. 
zelfallentscheidung (Buch III) auch die administrative Normsetzung (Buch II), die Verträge (Buch IV), die Amtshilfe (Buch V) und das behördliche Informationsmanagement (Buch VI) mit umfasst. Das VwVG beschränkt sich weitestgehend auf die Einzelfallentscheidung (sog. „Verfügung“). Andere Bereiche sind in der Schweiz entweder nicht einheitlich geregelt, wie beim verwaltungsrechtlichen Vertrag ${ }^{53}$. Oder die Normen finden sich in anderen Kodifikationen, etwa bezüglich administrative Normsetzung, Amtshilfe und behördliches Informationsmanagement ${ }^{54}$. Eine einheitliche Kodifikation erlaubt es, die für die Verwaltung maßgeblichen Strukturen und Prozesse der Informationsgewinnung, Entscheidungsfindung und Zusammenarbeit vor einem einheitlichen Reflexionsrahmen zu erfassen und kohärent zu regeln ${ }^{55}$.

Es ist aber nicht nur die gesamtheitliche Perspektive, welche Potential für Innovation bietet, sondern auch die einzelnen Regulierungsbereiche; der ReNEUAL-Musterentwurf nennt sie „Bücher“. Betrachtet man exemplarisch das Verfahren der Einzelfallentscheidung (Buch III) als „Kernbestand“ des Verwaltungsverfahrens, so dürfte das Innovationspotential indes eher begrenzt sein. In diesem Bereich wird besonders deutlich, dass das Verwaltungsverfahrensrecht „konkretisiertes Verfassungsrecht“"56 ist, steht doch die Verwirklichung der grundrechtlich abgesicherten Minimalgarantien (rechtliches Gehör, unparteiliche Entscheidfindung, Begründungspflicht, Beschleunigungsgebot) im Vordergrund. Aufgabe einer Kodifikation ist hier weniger das „ob“ einer Regelung als vielmehr das „,wie“ der Überführung in generell-abstrakte Normen und die verfahrenstechnische Umsetzung. Dennoch sind auch hier punktuelle Neuerungen denkbar, welche über die grundrechtlichen Minimalabsicherungen hinaus einen Beitrag zur „Bürgernähe“ der Verwaltung leisten können. Die Schaffung eines „Verantwortlichen Bediensteten“ bzw. „responsible official“ in Art. III-7 deutet in diese Richtung. Die Regelung, welche darauf abzielt, der Verwaltung ein persönlicheres „Gesicht“ und den betroffenen Privaten einen verlässlichen Ansprechpartner zu geben, ist grundsätzlich zu begrüßen ${ }^{57}$. Aus schweizerischer

53 Der Bund und die meisten Kantone kennen in den Verwaltungsverfahrensgesetzen keine Bestimmungen zum Vertrag im Verwaltungsrecht. Eine Ausnahme bildet das Verwaltungsrechtspflegegesetz des Kantons Glarus vom 4. Mai 1986 (Art. 123 ff.). Zudem bestehen zahlreiche Normen in den Sachgesetzen des besonderen Verwaltungsrechts (z.B. betreffend Konzessionsverträge, öffentlich-rechtliche Arbeitsverträge etc.).

54 Hierzu unten IV.2 und IV.3.

55 Schmidt-Aßmann, in: Hoffmann-Riem/ders. (Hrsg.), Verwaltungsverfahren und Verwaltungsverfahrensgesetz, 2002, S. 429 (432); Thurnherr, BJM, 2013, S. 217 (252).

56 Werner, „Verwaltungsrecht als konkretisiertes Verfassungsrecht“, DVBl 1959, S. $527 \mathrm{ff}$.

57 Zum Innovationsgehalt der vom italienischen Recht inspirierten Norm Schneider/Hofmann/Ziller (Hrsg.), ReNEUAL - Musterentwurf (FN 2), S. 75 Rn. 33. Eine vergleichbare Vorschrift wäre im Schweizer Recht indes schwer umsetzbar, da sich die Verantwortung in den zahlreich tätigen kollegialen Verwaltungsbehörden nicht ohne weiteres auf einen einzelnen „Bediensteten“ (recte „Mitglied“) übertragen lässt. Die 
und liechtensteinischer Sicht ist zu bedenken, ob nicht die Einführung alternativer Streitbeilegungsmechanismen einen zusätzlichen Beitrag zu einer „bürgernahen“ Verwaltung leisten könnte ${ }^{58}$. Die Kompromiss- und Konsenskultur ist in der kleinräumig organisierten Schweiz und in Liechtenstein traditionell sehr ausgeprägt und hat auch Niederschlag im Verfahrensrecht gefunden. $\mathrm{Zu}$ denken ist etwa an eine mündliche Schlichtungsverhandlung ${ }^{59}$ oder den Beizug einer außenstehenden Person als Mediatorin oder Mediator ${ }^{60}$. Vorläufig wurde im Musterentwurf auf eine entsprechende Regelung verzichtet $^{61}$, doch könnte dieses Anliegen in einem späteren Reformschritt verwirklicht werden ${ }^{62}$.

Die Kodifikation des EU-Verwaltungsverfahrensrechts bietet neben der wichtigen Aufgabe der Konsolidierung und Stabilisierung somit auch die Chance, schlummernde Steuerungsressourcen zu wecken. Doch in welchem Verhältnis sollen Nachführung und Innovation stehen? Der Entwurf kann bezüglich gesamtheitlicher Erfassung der Regelungsgegenstände sicher als innovativ gelten. Auch sind einzelne Bücher - etwa Buch VI zum behördlichen Informationsmanagement - schon per se von hohem Neuigkeitsgehalt, weil sie gewisse Bereiche erstmals in rechtlich geregelte und einheitliche Bahnen zu lenken versuchen. In anderen Büchern scheint der Innovationsgehalt hingegen eher punktuell zu sein. Aufgrund des schweizerischen Erfahrungshorizonts drängt sich die Frage auf, ob Nachführung und Innovation nicht stärker getrennt werden sollten. Sowohl im Verwaltungsverfahrensrecht wie im Verfassungsrecht wurde ein ,zweistufiges“ Vorgehen gewählt; in einem ersten Schritt war das Ziel die Nachführung und in weiteren Schritten wurden ganze Reformpakete (Verfassungsreform) oder punktuelle Neuerungen (Verwaltungsverfahren) vorangetrieben. Diese Etappierung erlaubte in einem ersten Schritt eine Beschränkung auf das Machbare und in einem zweiten Schritt eine bewusstere Reflexion über die Stoßrichtung und das Ausmaß

Idee einer einheitlichen Ansprechperson (unabhängig von einer allfälligen Entscheidverantwortung) wäre indes auch im schweizerischen Kontext sinnvoll.

58 Die gesetzliche Verankerung von alternativen Streitbeilegungsmöglichkeiten in einem allgemeinen Verwaltungsverfahrenserlass empfiehlt auch die Arbeitsgruppe für Verwaltungsrecht im Rechtsausschuss des Europäischen Parlaments (JURI): Vgl. Working Group on EU Administrative Law, Arbeitspapier vom 19. Oktober 2011 „State of Play and Future Prospects for EU Administrative Law“, Empfehlung Nr. 13, S. 31.

59 Art. 63 LVG sieht eine ,zwangslose Besprechung zur Herbeiführung einer Einigung“ vor, wenn „,mehrere Parteien mit widerstreitenden Rechten oder Interessen vor der Behörde auftreten".

60 Vgl. den 2005 eingefügten Art. $33 b$ VwVG zur gütlichen Einigung und Mediation sowie zahlreiche Schlichtungsverfahren in den Sachgesetzen des Besonderen Verwaltungsrechts (öffentliches Personalrecht, Datenschutzrecht etc.).

61 Schneider/Hofmann/Ziller (Hrsg.), ReNEUAL - Musterentwurf (FN 2), S. $107 \mathrm{f}$. Rn. 30. Vgl. indes das Streitschlichtungsverfahren zwischen beteiligten Behörden in Art. VI-31.

62 Vgl. auch Balthasar, in: Schneider/Rennert/Marsch (Hrsg.), ReNEUAL-Musterentwurf für ein EU-Verwaltungsverfahrensrecht, Tagungsband, 2016, S. 65 (86). 
der angestrebten Innovation. Die Problematik der schwierigen Suche nach dem „Kompromiss zwischen Ehrgeiz und notwendiger Selbstbeschränkung"63 ließe sich so zumindest teilweise entschärfen. Das Europäische Parlament hat mit seiner Entschließung vom 9. Juni 2016 diesen vorsichtig-pragmatischen Weg beschritten und konzentriert sich weitgehend auf eine Kodifizierung des Verfahrens der Einzelfallentscheidung, was in etwa dem Buch III des ReNEUAL-Entwurfs entspricht ${ }^{64}$. Die Leitidee ist ein „re-statement“, dessen Ziel die konsolidierte Aufarbeitung der bestehenden Gerichts-, Verwaltungs- und Ombudspraxis (,ombudsprudence“) ist ${ }^{65}$. Für das Wort „restatement" gibt es keine etablierte deutsche Übersetzung - der helvetisch geprägt Ausdruck „Nachführung“ scheint indes nicht unpassend.

\section{Regelungsgegenstand: \\ Wo beginnt und wo endet das ,Verwaltungsverfahren“?}

\section{Otto Mayers gemeinsames Erbe im deutschsprachigen Raum}

Aus schweizerischer, deutscher und österreichischer Sicht bestehen traditionell recht klare Vorstellungen darüber, was zum Verwaltungsverfahren gehört. Es ist das Verfahren der individuellen Entscheidfindung, an dessen Endpunkt ein Verwaltungsakt, ein Bescheid oder eine Verfügung steht ${ }^{66}$. Hintergrund dieser gemeinsamen Vorstellung ist das Erbe Otto Mayers (18461924) und der Juristischen Methode. Nach Mayer bedeutet Verwaltungsrecht „die rechtliche Ordnung der Verhältnisse zwischen dem verwaltenden Staate und dem ihm dabei begegnenden Untertanen “67. Und diese Verhältnisse soll-

63 Schneider/Hofmann/Ziller (Hrsg.), ReNEUAL - Musterentwurf (FN 2), S. 105 Rn. 13.

64 Vorschlag für eine Verordnung des Europäischen Parlaments und des Rates über das Verwaltungsverfahren der Organe, Einrichtungen, Ämter und Agenturen der Europäischen Union, PE573.120v01-00, 2016. Legislative Normsetzungsakte sind explizit ausgenommen (Art. 2 Ziff. 1 Bst. b); erfasst werden indes „Verwaltungsakte von allgemeiner Geltung“ (Art. 26). Zur Kritik an dieser nicht restlos klaren Eingrenzung des Regelungsgegenstands unten FN 105.

65 Galetta/Hofmann/Mir Puigpelat/Ziller, The context and legal elements of a Proposal for a Regulation on the Administrative Procedure of the European Union's institutions, bodies, offices and agencies, European Parliament's Policy Department for Citizens' Rights and Constitutional Affairs, PE 536.487, 2016, S. 14. Vgl. auch die Definition in Ziller, Alternatives in Drafting an EU Administrative Procedure Law, European Parliament's Policy Department for Citizens' Rights and Constitutional Affairs, PE 462.417, 2011, S. 17.

66 Das deutsche VwVfG erfasst - als jüngste der drei Kodifikationen - in den $\S \S 54$ ff. auch den öffentlich-rechtlichen Vertrag. In der Schweiz regelt nur ein Kanton den Vertrag in seinem Verwaltungsrechtspflegegesetz (vgl. Hinweis in FN 53). Dem liechtensteinischen LVG liegt demgegenüber stärker der Ansatz einer umfassenden Kodifikation zu Grunde (vgl. FN 52).

67 Mayer, Verwaltungsrecht (FN 47), S. 13. 
ten geprägt sein von „,tunlichster Justizförmigkeit“68. Die damit verbundene starke Fokussierung war wohl das Erfolgsrezept der Juristischen Methode zugleich aber ihre größte Schwäche ${ }^{69}$. Zahlreiche Bereiche der Verwaltungstätigkeit, etwa verwaltungsinterne Handlungen, Beziehungen zwischen Verwaltungsbehörden, nicht-förmliches Verwaltungshandeln („Realakte“), rechtsetzende Verwaltungstätigkeit und Kooperationen zwischen Verwaltung und Privaten entschwanden aus dem Fokus der Verwaltungsrechtswissenschaft. Diese Defizite stellte Peter Saladin (1935-1997) bereits 1979 für die Schweiz fest und forderte ein breiteres Verfahrensverständnis, welches auch die verwaltungsrechtlichen Verträge und die administrative Normsetzung mit einschließen müsse. Zudem forderte er eine Klärung, inwiefern das Verwaltungsverfahrensrecht auch auf Private Anwendung finde, welche öffentliche Aufgaben wahrnehmen ${ }^{70}$. Auch die „Neue Verwaltungsrechtswissenschaft“, welche sich in einem umfassenderen Sinn als Steuerungswissenschaft versteht, will diese Defizite ausgleichen ${ }^{71}$. Im ReNEUAL-Musterentwurf, der neben dem Verfahren der individuellen Entscheidfindung (Buch III) auch die administrative Normsetzung (Buch II), die Verträge (Buch IV), die Amtshilfe (Buch V) und das behördliche Informationsmanagement (Buch VI) kodifiziert, schlägt sich diese Absicht deutlich nieder ${ }^{72}$. Es wäre indes zu einfach, ein enges bzw. breites Verfahrensverständnis auf den Gegensatz zwischen „Juristischer Methode“ einerseits und „Neuer Verwaltungsrechtswissenschaft" andererseits zu reduzieren. Die Frage, was aus Sicht der bestehenden europäischen Rechtsordnungen zum Verwaltungsverfahrensrecht gehört oder nicht, ist auch von weiteren Faktoren abhängig. Ich möchte dies aus schweizerischer Perspektive an drei Beispiele verdeutlichen:

\section{Administrative Normsetzung (Buch II)}

Die administrative Normsetzung gehört nach traditionellem Verständnis aller deutschsprachigen Staaten nicht zum Verwaltungsverfahren - im Unterschied etwa zu Spanien ${ }^{73}$ oder den $\mathrm{USA}^{74}$. In Deutschland und Österreich be-

68 Mayer, Verwaltungsrecht (FN 47), S. 62.

69 Pünder, in: Allgemeines Verwaltungsrecht (FN 38), S. 408 f. Rn. 3.

70 Saladin, Das Verwaltungsverfahrensrecht des Bundes, 1979, S. 25 ff.

71 Vgl. exemplarisch Schmidt-Aßmann, in: Verwaltungsverfahren und Verwaltungsverfahrensgesetz (FN 55), S. 432 ff.; ders., in: Hoffmann-Riem/Schmidt-Aßmann/ Voßkuhle (Hrsg.), Grundlagen des Verwaltungsrechts, Bd. II, 2. Aufl. 2012, S. 495 (522 ff. Rn. 48 ff.).

72 Schneider/Hofmann/Ziller (Hrsg.), ReNEUAL - Musterentwurf (FN 2), S. 9 f. Rn. 32 .

73 Mir Puigpelat, DÖV 2006, S. 841 ff.; Montoro-Chiner, in: Hill/Pitschas (Hrsg.), Europäisches Verwaltungsrecht, 2004, S. $127 \mathrm{ff}$.

74 Pünder, Exekutive Normsetzung in den Vereinigten Staaten von Amerika und der Bundesrepublik Deutschland, 1995, S. 118 f. 
stehen bislang keine entsprechenden Kodifikationen in allgemeiner Form ${ }^{75}$. Dies ist anders in der Schweiz. Die Konsultation und Partizipation betroffener Kreise (Kantone, Gemeinden, politische Parteien, Interessengruppierungen) sowie einer breiteren Öffentlichkeit ist auf Bundesebene (und teilweise auch in den Kantonen ${ }^{76}$ ) verfassungsrechtlich verankert und gesetzlich geregelt ${ }^{77}$. Demnach unterliegen die von Regierung und Verwaltung erlassenen Verordnungen einer „Vernehmlassung“, wenn sie „von großer politischer, finanzieller, wirtschaftlicher, ökologischer, sozialer oder kultureller Tragweite sind“ oder wenn sie „einzelne oder alle Kantone in erheblichem Maß betreffen oder in erheblichem Maß außerhalb der Bundesverwaltung vollzogen werden“78. „Vernehmlassung“ bedeutet, dass die vorbereitenden Unterlagen des Rechtsetzungsprojekts (i. d. R. ein Norm-Entwurf mit einem erläuternden Bericht) elektronisch publiziert werden und darüber hinaus bestimmte Kreise gezielt zur Stellungnahme eingeladen werden. Dabei wird eine Frist von mindestens drei Monaten angesetzt. Die eingegangenen Stellungnahmen (,Vernehmlassungen “) werden zur Kenntnis genommen, gewichtet, ausgewertet und in einem Bericht zusammengefasst. Die Unterlagen der Vernehmlassung (Bericht, Stellungnahmen, Protokolle allfälliger Anhörungen) werden anschließend veröffentlicht ${ }^{79}$. Dieses Verfahren weist offensichtliche Parallelen zur Ausgestaltung des Verfahrens im ReNEUAL-Musterentwurf (Buch II) auf. Auch die Motive, die hinter dem Vernehmlassungsverfahren stehen, gleichen den Absichten des Musterentwurfs. Beiden Verfahren liegt ein partizipatives Demokratieverständnis zu Grunde und die Absicht, die Transparenz und Akzeptanz des Normsetzungsverfahrens zu erhöhen ${ }^{80}$. Eine besondere Rolle spielt im schweizerischen Vernehmlassungsverfahren zudem das Abtasten der Vollzugstauglichkeit im Verwaltungsverbund von Bund, Kantonen und Gemeinden ${ }^{81}$. Das Verfahren trägt somit nicht zur demokratischen Legitimation bei, sondern dient auch der Funktionsfähigkeit eines ausgeprägt föderalistischen Vollzugsregimes $^{82}$.

75 Vgl. Pünder, Exekutive Normsetzung (FN 74), S. 140; Schmidt-Aßmann, in: Grundlagen des Verwaltungsrechts II (FN 71), S. 504 Rn. 13.

76 In gewissen Kantonen ist das Recht der Partizipation im Rahmen der Vernehmlassung als verfassungsmäßiges Recht ausgestaltet. Vgl. etwa $§ 27$ der Verfassung des Kantons Luzern vom 17. Juni 2007. Eine besonders ausgeprägte Form der direkten Bürgerpartizipation kennt der Kanton Appenzell Außerrhoden mit der Volksdiskussion: Art. 56 der Verfassung des Kantons Appenzell Außerrhoden vom 30. April 1995.

77 Art. 147 BV; Bundesgesetz über das Vernehmlassungsverfahren vom 18. März 2005 (Vernehmlassungsgesetz, SR 172.061).

78 Art. 3 Abs. 1 Bst. d und e Vernehmlassungsgesetz.

79 Vgl. für das Verfahren im Einzelnen Art. 5-9 Vernehmlassungsgesetz.

80 Schneider/Hofmann/Ziller (Hrsg.), ReNEUAL - Musterentwurf (FN 2), S. 32 Rn. 1; Art. 2 Vernehmlassungsgesetz.

81 Art. 2 Abs. 2 Vernehmlassungsgesetz.

82 Martenet, Architecture des pouvoirs: Enjeux et perspectives pour un État, une union des États et les Nations Unies, 2016, S. 49. 
Die Parallelen zwischen der geltenden Regelung in der Schweiz und der vom ReNEUAL-Musterentwurf vorgeschlagenen Lösung für die EU sind somit erstaunlich, obwohl der Entwurf in erster Linie vom US-amerikanischen Vorbild geprägt wurde ${ }^{83}$. Ein Unterschied besteht möglicherweise insofern, als die „Vernehmlassung“ in der Schweiz als primär demokratisch-föderalistisch motiviertes Entscheidungsverfahren verstanden wird. Als Folge der direktdemokratischen Mitwirkungsrechte in der Gesetzgebung und der starken Stellung der Kantone beim Normvollzug ist die frühzeitige Sicherstellung der Akzeptanz von Gesetzesentwürfen in der Schweiz von überragender Bedeutung. Es mag auf den ersten Blick paradox erscheinen, doch fördert gerade die direkte Mitsprache des Volkes bei der Gesetzgebung verhandlungsdemokratische Elemente, welche exemplarisch im Vernehmlassungsverfahren zum Ausdruck kommen ${ }^{84}$. Diese Kultur der „Vernehmlassung“, strahlt auch auf Bereiche aus, wo Regierung und Verwaltung in eigener Kompetenz rechtsetzend tätig sind. Demokratische Legitimation wird in der Schweiz insofern stärker prozedural - durch die oft mühsame und zeitraubende Suche nach einem Konsens und Kompromiss - definiert ${ }^{85}$. Kantone, Gemeinden, politische Parteien, Wirtschaftsverbände und NGOs übernehmen in diesem Verhandlungsprozess eine wichtige Rolle, indem sie zur Qualität der Vernehmlassung beitragen und der Bevölkerung Orientierung geben ${ }^{86}$. Hier scheint ein Unterschied zu gewissen Mitgliedstaaten der EU zu bestehen, welche demokratische Legitimation in erster Linie mit einem bestimmten Staatsorgan (Parlament) und seinen Akten (Gesetzen) identifizieren ${ }^{87}$. Die Einflussnahme von Interessengruppen auf die administrative Normsetzung wird dann weniger als Gewinn an demokratischer Partizipation wahrgenommen, sondern vielmehr als Gefahr für die Demokratie, weil dem durch das Parlament im Gesetz bekundeten demokratischen Willen in seiner Umsetzung durch Lobbyismus und Korporatismus eine Verfälschung droht ${ }^{88}$. Möglicher-

83 Zur amerikanischen Rezeptionsvorlage Ruffert, in: ReNEUAL-Musterentwurf für ein EU-Verwaltungsverfahrensrecht. Tagungsband (FN 62), S. 111 (114 ff.); Schneider/Hofmann/Ziller (Hrsg.), ReNEUAL - Musterentwurf (FN 2), S. 40 Rn. 23.

84 Armingeon, LeGes - Gesetzgebung \& Evaluation, 2004/3, S. 59 ff.; Linder, Schweizerische Demokratie, 3. Aufl. 2012, S. $331 \mathrm{f}$.

85 Armingeon, LeGes - Gesetzgebung \& Evaluation, 2004/3, S. 59 f.; Linder, Schweizerische Demokratie (FN 84), S. 341 ff.; J.P. Müller, Demokratische Gerechtigkeit 1993, S. 148 ff.

86 Armingeon, LeGes - Gesetzgebung \& Evaluation, 2004/3, S. 59 (67); J.P. Müller, Demokratische Gerechtigkeit (FN 85), S. 193 ff.

87 Nach deutschem Verständnis wird die demokratische Legitimation exekutiver Normsetzung in erster Linie durch die vom Gesetzgeber erfolgte Ermächtigung erzeugt: Pünder, Exekutive Normsetzung (FN 74), S. 92 f.; Wallrabenstein, in: ReNEUAL-Musterentwurf für ein EU-Verwaltungsverfahrensrecht. Tagungsband (FN 62), S. 118 (124). Zu einem auch in Deutschland zunehmend partizipativen Demokratieverständnis Pünder, in: Allgemeines Verwaltungsrecht (FN 38), S. $420 \mathrm{f}$. Rn. 17.

88 Vgl. insb. die entsprechenden Bedenken aus deutscher Perspektive: Ruffert, in: ReNEUAL-Musterentwurf für ein EU-Verwaltungsverfahrensrecht, Tagungsband (FN 62), S. 123. 
weise passt indes das prozedurale und stark föderalistisch geprägte Demokratieverständnis im Mehrebenensystem Schweiz besser zur EU als eine dichotomisch anmutende Unterscheidung zwischen parlamentarischem Gesetzgebungsverfahren und exekutivem Vollzug ${ }^{89}$.

Aus schweizerischer Sicht ließe sich trotz dieser großen Gemeinsamkeiten kritisch fragen, ob die Konsultation und Partizipation im Vorfeld der exekutiven Normsetzung kodifikatorisch mit dem Verfahren der individuellen Entscheidfindung ,unter einem Dach“ vereinigt werden soll. Zwar gibt es Berührungspunkte, etwa dort, wo Großverwaltungsakte die Konsultation einer breiteren Öffentlichkeit angezeigt erscheinen lassen ${ }^{90}$. Vergleichend betrachtet erscheinen die Verfahren aber dennoch unterschiedliche Ausprägungen aufzuweisen ${ }^{91}$ : Das Verfahren administrativer Normsetzung weist eine systematische Nähe zur legislativen Normsetzung auf und ist stärker demokratisch-föderalistisch als rechtsstaatlich geprägt ${ }^{92}$. Verfahrensziel ist ein politischer Konsens oder Kompromiss, aber auch das Abtasten von Vollzugswiderständen im Verwaltungsverbund. Maßstab ist daher eine möglichst breite und transparente Beteiligung aller interessierten Akteure. Ziel des auf individuelle Entscheidungen ausgerichteten Verwaltungsverfahrens ist hingegen eine inhaltlich richtige, faire und rasche Entscheidung. Maßstab sind die aus den Verfahrensgrundrechten der unmittelbar Betroffenen abgeleiteten rechtsstaatlichen Standards. Vielfach ist das individuelle Verwaltungsverfahren zudem Vorstufe zu einem verwaltungsinternen oder gerichtlichen Beschwerdeverfahren.

\section{Fragen des Verwaltungsverbunds und der Verwaltungskooperation (Buch V und VI)}

Betrachtet man die enorme Bedeutung der Verbundverwaltung im Mehrebenensystem der Schweiz, dann erstaunt die nahezu vollständige „Föderalismusblindheit" des schweizerischen Verwaltungsverfahrensrechts. Fragen der Amtshilfe und des Informationsaustauschs - innerschweizerisch wie international - werden im VwVG nicht geregelt ${ }^{93}$. Das Institut der Amtshilfe ist

89 Vgl. Blondel, in: Democracy in the European Union - A Contribution from the Swiss Perspective (FN 17), S. 73, 85; Martenet (FN 82), S. 285 ff. Zu den Grenzen der Übertragbarkeit nationaler Demokratiemodelle auf die EU: Oesch, Europarecht (FN 4), Rn. 510.

90 Vgl. hierzu Art. III-25 ReNEUAL-Musterentwurf, der inhaltlich enge Bezüge zum Buch II aufweist, ohne indes explizit auf die entsprechenden Bestimmungen zu verweisen.

91 Hierbei handelt es sich indes eher um eine typologische Ausdifferenzierung und nicht um eine kategoriale Unterscheidung. Administrative Normsetzung und Normanwendung sind sich strukturell verwandt: Schindler, Verwaltungsermessen, 2010, Rn. 445.

92 Wallrabenstein in: ReNEUAL-Musterentwurf für ein EU-Verwaltungsverfahrensrecht, Tagungsband (FN 62), S. 124.

93 Lediglich in Art. 43 VwVG findet sich eine Bestimmung zur Vollstreckungshilfe. 
in der schweizerischen Lehre und Rechtsprechung zwar seit langem anerkannt. Als Ausfluss der „Bundestreue“ verpflichtet die Bundesverfassung Bund und Kantone in grundsätzlicher Weise, einander Rechts- und Amtshilfe zu leisten ${ }^{94}$. Was darunter im Einzelnen zu verstehen ist, ergibt sich indes aus der Praxis und vereinzelten Bestimmungen, die mehrheitlich im Verwaltungsorganisationsrecht oder in Spezialgesetzen zu finden sind. Detaillierte Regelungen über die Amtshilfe bestehen vielfach nur für diejenigen Fälle, in denen die Rechtsposition des Einzelnen durch eine Amtshilfehandlung beeinträchtigt zu werden droht, etwa im Bereich des Steuerrechts oder des Datenschutzrechts ${ }^{95}$. Ansonsten sind die entsprechenden Vorschriften sehr offen und allgemein gehalten. Dies kann mit Blick auf einen bereits lange bestehenden und leidlich funktionierenden Verwaltungsverbund wie die Schweiz sinnvoll sein, auch wenn aus Sicht der betroffenen Individuen präzisere und transparentere Regeln der Amtshilfe zu begrüßen wären ${ }^{96}$. Aus schweizerischer Sicht erscheinen die Bücher V und VI daher als wertvolle Inspirationsquelle, wenn es darum geht, die eigenen Spielregeln im Verwaltungsverbund zu reflektieren, zu strukturieren und transparent zu machen.

\section{Rechtsbehelfe und gerichtlicher Rechtsschutz}

Die meisten schweizerischen Kodifikationen des Verwaltungsverfahrens dies sind neben dem VwVG auch die kantonalen Verwaltungsrechtspflegegesetze - regeln im gleichen Erlass nicht nur das erstinstanzliche und nicht-streitige Verwaltungsverfahren, sondern darüber hinaus auch das verwaltungsinterne Beschwerdeverfahren und das verwaltungsgerichtliche Verfahren ${ }^{97}$. Dasselbe kodifikatorische Grundkonzept liegt auch dem liechtensteinischen LVG zugrunde ${ }^{98}$. Diese kodifikatorische Zusammenführung ist historisch bedingt, sollte das Verwaltungsverfahren doch möglichst ,justizförmig“ ausgestaltet sein $^{99}$. Zudem erfolgte die Kodifikation des Verwaltungsverfahrens in der Schweiz und Liechtenstein - im Gegensatz zu Deutschland und Österreich $^{100}$ - meist zeitgleich mit dem Auf- und Ausbau der Verwaltungsgerichts-

94 Art. 44 Abs. 2 BV.

95 Vgl. eine überblicksartige Zusammenstellung der verstreuten Spezialbestimmungen bei Kiener/Rütsche/Kuhn, Öffentliches Verfahrensrecht, 2. Aufl. 2015, Rn. 1153 ff.

96 Zum gesetzgeberischen Konkretisierungsbedarf: Schweizer, in: Ehrenzeller/ Schindler/Schweizer/Vallender (Hrsg.), Die schweizerische Bundesverfassung, St. Galler Kommentar, 3. Aufl. 2014, Art. 44, Rn. 32.

97 Das VwVG selber regelte ursprünglich nur das verwaltungsinterne Beschwerdeverfahren. Durch einen Verweis in Art. 37 des Bundesgesetzes über das Bundesverwaltungsgericht vom 17. Juni 2005 (SR 173.32) findet das VwVG aber - mit wenigen Ausnahmen - auch auf das Verfahren vor dem Bundesverwaltungsgericht Anwendung.

98 Vgl. Kley, Grundriss des liechtensteinischen Verwaltungsrechts, 1998, S. 275 ff.

99 Vgl. oben bei FN 68.

100 In Österreich wurde der Verwaltungsgerichtshof 1876 geschaffen, während die Kodifikation des Verfahrens 1925 erfolgte. In den deutschen Ländern erfolgte die 
barkeit; diese sollte „einen verfahrensmäßig durchgeformten und gesicherten Unterbau “101 in Form eines geregelten Verwaltungsverfahrens erhalten. Diese - unter einer vertikalen Perspektive - wesentlich umfassenderen Kodifikationen haben zudem den Vorteil einer ganzheitlichen Regelung des „Rechtsschutzes", der nicht erst mit der gerichtlichen Überprüfung beginnt, sondern beim ersten Kontakt zwischen Verwaltung und Individuum einsetzt. Schließlich bestehen bei einer Zusammenfassung der gesamten „Verwaltungsrechtspflege“ in einem Gesetz Harmonisierungs- und Synergieeffekte, indem sich gewisse Regeln - etwa zum Parteibegriff, zur Fristenberechnung, zu den Beweismitteln oder zur Begründungspflicht - in einem Allgemeinen Teil „,vor die Klammer ziehen lassen". Kehrseite dieser integralen Sichtweise ist in der Schweiz aber die Engführung des Verwaltungsverfahrens auf das Verfahren der individuellen Entscheidfindung. Verwaltungsverfahren, verwaltungsinternes Beschwerdeverfahren und verwaltungsgerichtliches Verfahren sind gleichsam verschiedene zeitliche Schritte auf demselben (engen) Pfad der Rechtsverwirklichung ${ }^{102}$. Zudem lässt diese Sichtweise allfällige Unterschiede zwischen verwaltungsinternem und verwaltungsgerichtlichem Verfahren in den Hintergrund treten und widerspricht so dem Trennungsprinzip, wie es etwa für Deutschland postuliert wird ${ }^{103}$. Wird der Gegenstand „Verwaltungsverfahren“ bewusst breit verstanden, wie dies im ReNEUAL-Musterentwurf zum Ausdruck kommt, dann kann eine Entflechtung von Verwaltungsverfahren und Verwaltungsprozess sachgerechter sein ${ }^{104}$.

Nachdem sich das Europäische Parlament nun für eine schlanke Kodifikation entschieden hat, welche das individuelle Entscheidverfahren in den Vordergrund rück $\mathrm{t}^{105}$, stellt sich die Frage, ob nicht zumindest eine minimale Kodifizierung der verwaltungsinternen Rechtsschutzmöglichkeiten sinnvoll wäre ${ }^{106}$. Diese Frage stellt sich umso drängender, als der Entwurf des Parlaments in Art. 20 Abs. 2 einen Anspruch auf ,administrative review “107 statu-

Kodifikation in den Ländern mehrheitlich nach Einführung der Verwaltungsgerichtsbarkeit: Pünder, in: Allgemeines Verwaltungsrecht (FN 38), S. 409 ff. Rn. 4 f.

101 Imboden, Diskussionsvotum, VVDStRL 17 (1958), S. 214. Für Liechtenstein: Schädler, Arbeitspapiere Liechtenstein-Institut, 54, 2016, S. 22.

102 Schindler, in: Kommentar zum Bundesgesetz über das Verwaltungsverfahren (FN 29), Einleitung Rn. 23; Thurnherr, BJM, 2013, S.217 (252).

103 Schmidt-Aßmann, Das allgemeine Verwaltungsrecht als Ordnungsidee, 2. Aufl. 2004, S. 374, Rn. 173. Vgl. aber zur auch in Deutschland anerkannten Konnexität und Komplementarität der Verfahren: Jochum, Verwaltungsverfahrensrecht und Verwaltungsprozeßrecht (FN 49).

104 Thurnherr, BJM, 2013, S. 217 (252).

105 Der Anwendungsbereich des Entwurfs ist leider nicht mit ausreichender Präzision umschrieben. Vgl. die Kritik bei Schneider, in: ReNEUAL-Musterentwurf für ein EU-Verwaltungsverfahrensrecht. Tagungsband (FN 62), S. 129 (134 f.).

106 In diesem Sinne auch Bermann, A Restatement of European Administrative Law: Problems and Prospects, 2009, S. 8 f.

107 Die deutsche Übersetzung („verwaltungsrechtliche Überprüfung“) ist irreführend, da sich „,administrative review“ (im Unterschied zu ,judicial review“) auf die 
iert und sich nicht darauf beschränkt, eine bloße Rechtsbehelfsbelehrung vorzusehen, wie dies noch der ReNEUAL-Musterentwurf tat (Art. III-30). Gegenwärtig ist der verwaltungsinterne Rechtsschutz im Bereich der EU-Eigenverwaltung sehr unterschiedlich ausgestaltet. Im Bereich des Verwaltungshandelns der EU-Kommission besteht kein förmliches verwaltungsinternes Rechtsschutzverfahren, wohl aber teilweise im Bereich der EU-Agenturen. Letztere unterscheiden sich stark. Das verwaltungsinterne Rechtsschutzverfahren in der EU-Eigenverwaltung ist damit nicht nur durch eine „Sektoralisierung des Rechtsschutzes im Bereich der Agenturen [...], sondern darüber hinaus [durch] divergente Rechtsschutzmöglichkeiten gegenüber dem Verwaltungshandeln der Agenturen auf der einen und dem Verwaltungshandeln der Kommission auf der anderen Seite " ${ }^{108}$ gekennzeichnet. Angesichts dieses Befunds wurde die Forderung nach Einführung eines verwaltungsinternen Rechtsschutzverfahrens zur „Wiederherstellung verlorengegangener Rechtsschutzkohärenz" auch für den Bereich des Verwaltungshandelns der EU-Kommission erhoben ${ }^{109}$. Die Kompetenz zur Ausdehnung des Anwendungsbereichs einer entsprechenden Verordnung würde durchaus bestehen $^{110}$.

Die drei hier beschriebenen Beispiele machen deutlich, dass der ReNEUAL-Musterentwurf eine wichtige und überzeugende Reformagenda aufzeigt. An dieser Reformagenda können sich auch Nicht-Mitgliedstaaten wie die Schweiz orientieren, was der Bereich Verwaltungskooperation deutlich macht. Daneben bestehen aber auch außerhalb des Musterentwurfs „Verwaltungsverfahren", welche einer vereinheitlichenden Kodifikation harren, etwa der gesamte Bereich des verwaltungsinternen Rechtsschutzes. Schließlich zeigen die drei ausgewählten Aspekte, dass kein zwingender Zusammenhang zwischen Reformbedarf und dem Bedürfnis nach einer umfassenden und einheitlichen Kodifikation besteht. Das Verfahren der administrativen Normsetzung macht exemplarisch deutlich, dass eine Kodifikation außerhalb des Regelungsgegenstandes Verwaltungsverfahren möglich und vermutlich auch sachgerechter ist. Eine Modularisierung der Reformagenda in verschiedene Kodifikationsprojekte würde eine Ausdifferenzierung erlauben, welche sich noch stärker an den unterschiedlichen Zielen und Qualitätskriterien der verschiedenen Verfahrenstypen orientiert. Vermutlich dürften mehrere kleinere Kodifikationsprojekte im politisch-administrativen Umfeld auch eher Verwirklichungschancen haben als eine ambitionierte „Großkodifikation“. Der Umstand, dass das Europäische Parlament mit seinem Entwurf vorerst eine vergleichsweise schmal konzipierte Kodifikation des individuellen Entscheidungsverfahrens anstrebt, scheint diese Vermutung zu

Kontrollinstanz und nicht auf den inhaltlichen Kontrollmaßstab (Verwaltungsrecht) bezieht.

108 Streckert, Verwaltungsinterner Unionsrechtsschutz, Kohärenter Rechtsschutz durch Einführung eines Widerspruchskammermodells für die Europäische Kommission, 2016, S. 50.

109 Streckert, Verwaltungsinterner Unionsrechtsschutz (FN 108), S. 86.

110 Streckert, Verwaltungsinterner Unionsrechtsschutz (FN 108), S. 96. 
bestätigen. Es zeigt aber auch die Stärke des ReNEUAL-Musterentwurfs, der mit seinen einzelnen „Büchern“ bereits „Sollbruchstellen“ markiert, welche eine gestaffelte Verwirklichung des Reformprojekts erlaubt ${ }^{111}$.

\section{Regelungsstruktur und -sprache: Präzision, Kürze, Einfachheit}

Aus schweizerischer Sicht ist die Lektüre des ReNEUAL-Musterentwurfs gewöhnungsbedürftig. Der Grund hierfür liegt darin, dass Normtexte in der Schweiz erheblich schlanker und stärker durchstrukturiert sind. Im Idealfall orientieren sich Gesetze an der auf den Schöpfer des Zivilgesetzbuchs zurückgehenden „Eugen-Huber-Regel“: „Pro Artikel höchstens drei Absätze. Pro Absatz ein Satz. Pro Satz ein Gedanke“112. Dieser Regel folgt der Musterentwurf nicht. Freilich ist dies keine Besonderheit des Musterentwurfs, sondern ein genereller Unterschied zwischen der in der Schweiz üblichen Rechtsetzungstechnik und derjenigen in der $\mathrm{EU}^{113}$. Und die Schweiz kann sich diesem EU-Stil auch nicht ganz entziehen. Im Bereich des autonomen Nachvollzugs von EU-Recht hat sich auch im Schweizer Recht der Geist Eugen Hubers weitgehend verflüchtigt.

Eng mit der Normstruktur ist die Sprache verbunden. Gewiss ist eine getreue Umsetzung der „Eugen-Huber-Regel“ im Rahmen der europäischen Gesetzgebung (ungleich) schwieriger als in der nationalen Gesetzgebung. Der Zwang zur Konsensbildung über die Landesgrenzen und Rechtskulturen hinweg und die fehlende gemeinsame Begrifflichkeit können schlanken Formulierungen im Wege stehen ${ }^{114}$. Vor diesem Hintergrund erscheint es weder sinnvoll noch praktikabel, die „Eugen-Huber-Regel“ bei der Erarbeitung des ReNEUAL-Musterentwurfs als unbedingt einzuhaltende Maxime der Gesetzesredaktion zu verstehen. Sie soll aber als Richtschnur dafür dienen, dass bei der Gliederung des Musterentwurfs möglichst einfache Einheiten zu bilden sind, welche die Gedankenabfolge der Regelung möglichst direkt in der Normstruktur wiedergeben ${ }^{115}$.

111 Vgl. Schneider, in: ReNEUAL-Musterentwurf für ein EU-Verwaltungsverfahrensrecht. Tagungsband (FN 62), S. 3 (18).

112 Huber, Erläuterungen zum Vorentwurf eines schweizerischen Zivilgesetzbuches, Erster Band, 1914, S. 14; Bundesamt für Justiz (Hrsg.), Gesetzgebungsleitfaden, 3. Aufl. 2007, Rn. 887; Müller/Uhlmann, Elemente einer Rechtsetzungslehre, 3. Aufl. 2013, Rn. 332.

113 Vgl. zur Qualität von EU-Gesetzgebungsakten aus schweizerischer Sicht: Jaag/ Hänni, Europarecht (FN 3), Rn. 226; Müller/Uhlmann, Rechtsetzungslehre (FN 112).

114 Vgl. Müller/Uhlmann, Rechtsetzungslehre (FN 112), Rn. 329 sowie Kuhn, LeGes - Gesetzgebung \& Evaluation, 1999/3, S. 49 ff.

115 Vgl. Lötscher, LeGes - Gesetzgebung \& Evaluation, 1994/3, S. 71; in diesem Sinn auch die Empfehlung Nr. 20 der Working Group on EU Administrative Law, Arbeitspapier (FN 58), S. 32. 
Voraussetzung einer schlanken Normstruktur nach Maßgabe der „EugenHuber-Regel“ ist eine konzise und leicht verständliche Gesetzessprache. Normen sollen demnach möglichst präzise, kurz und einfach formuliert werden $^{116}$. Präzision meint prägnante Formulierung, die klar definierte Begriffe verwendet und den normativen Kern deutlich erkennbar macht. Kürze bedeutet Beschränkung auf das normativ Wesentliche. Erläuterungen, sprachliche Ausschmückungen und Redundanzen im Gesetzestext sind in der Regel zu vermeiden. Einfachheit verlangt nach einem übersichtlichen Satzbau und einem leicht verständlichen sprachlichen Ausdruck. Auf Substantivierungen, Fremdwörter und Fachausdrücke ist nach Möglichkeit zu verzichten.

Wendet man die hier genannten Kriterien auf den vorliegenden ReNEUALMusterentwurf an, so besteht Potential für Verbesserung. Auffallend ist etwa, dass der Vorbehalt zu Gunsten sektorspezifischer Verfahrensvorschriften im EU-Recht zwar in allgemeiner Form in Art. I-2 genannt wird. Entsprechende Vorbehalte folgen dann aber in den einzelnen Büchern wieder an verschiedensten Orten. Wenn der Allgemeine Teil aber dazu dienen soll, „vor die Klammer zu ziehen", dann sind derartige Wiederholungen in den einzelnen Büchern normativ gesehen überflüssig und erschweren die Lesbarkeit unnötig. Exemplarisch lässt sich dies am Beispiel von Art. III-9 zu den Fristen für den Verfahrensabschluss zeigen. ${ }^{117}$ Dass sich diese Norm sprachlich kürzen und vereinfachen lässt, soll am folgenden Beispiel gezeigt werden:

III-9 Fristen für den Verfahrensabschluss

(1) Die Behörde erlässt die Entscheidung unverzüglich, spätestens aber innerhalb von drei Monaten.

(2) Die Frist beginnt mit Eingang der vollständigen Antragsunterlagen oder zu dem Zeitpunkt, in dem das Verfahren von Amts wegen eingeleitet wird.

(3) Ist der Verfahrensabschluss innerhalb der ordentlichen Frist aufgrund der Komplexität des Einzelfalls oder anderer Hindernisse nicht möglich, teilt die Behörde den Parteien die Gründe der Verfahrensverlängerung

116 Müller/Uhlmann, Rechtsetzungslehre (FN 112), Rn. $332 \mathrm{ff}$.

117 Vgl. den Originaltext des Musterentwurfs:

(1) ${ }^{1}$ Die Behörde erlässt die Entscheidung unverzüglich und innerhalb eines angemessenen Zeitraums. ${ }^{2}$ Fristen werden durch das sektorspezifische EU-Recht festgelegt. Sehen sektorspezifische Verfahrensvorschriften keine Fristen vor, beträgt die Verfahrensfrist drei Monate.

(2) Die Frist beginnt mit Eingang der vollständigen Antragsunterlagen oder zu dem Zeitpunkt, in dem das Verfahren von Amts wegen eingeleitet wird.

(3) ${ }^{1}$ Stehen die Komplexität des Einzelfalls oder andere Hindernisse einer Beurteilung des Falls innerhalb der Frist entgegen, sind die Parteien darüber zu informieren und die Entscheidung ist unverzüglich zu erlassen. ${ }^{2}$ Die Behörde informiert die Parteien schriftlich über die Gründe der Verfahrensverlängerung und teilt diesen, soweit möglich, den voraussichtlichen Zeitpunkt mit, in dem die Entscheidung erlassen wird. ${ }^{3}$ Durch sektorspezifisches EU-Recht vorgesehene Einschränkungen für eine Verlängerung von Verfahren bleiben vorbehalten.

(4) Das sektorspezifische EU-Recht legt die Rechtsfolgen einer Fristverletzung fest. 
und den voraussichtlichen Zeitpunkt der Verfahrenserledigung unverzüglich und schriftlich mit.

\section{Regelungsdichte: Balanceakt zwischen Bestimmtheit und Offenheit}

In der Diskussion des ReNEUAL-Musterentwurfs wurde die Frage nach der angemessenen Bestimmtheit verfahrensrechtlicher Regelungen bereits verschiedentlich aufgeworfen. Ingo Kraft plädiert aus verfahrensökonomischen Überlegungen für eine weitgehende Vermeidung offener Rechtsbegrif$\mathrm{fe}^{118}$. Jens-Peter Schneider betont, dass verfahrensrechtliche Kodifikationen im Interesse ihrer Flexibilität für neue Regelungssituationen in gewissem Ausmaß nicht auf unbestimmte Rechtsbegriffe verzichten können ${ }^{119}$. Michael Fehling vertritt die Ansicht, der Musterentwurf wahre eine gute Balance zwischen rechtsstaatlichen Formalisierungsnotwendigkeiten einerseits und Flexibilitätsinteressen der Verwaltung andererseits ${ }^{120}$.

Der angemessene Bestimmtheitsgrad einer Norm lässt sich kaum generell festgelegen, sondern ist grundsätzlich für jede einzelne Norm im Rahmen der gesetzgeberischen Interessenabwägung zu optimieren ${ }^{121}$. Allerdings fällt auf, dass das materielle Verwaltungsrecht wesentlich stärker durch offene Normen und Ermessensspielräume geprägt ist als das Verfahrensrecht ${ }^{122}$. Dieser Befund ergibt sich im Wesentlichen aus den unterschiedlichen Funktionen des formellen und materiellen Verwaltungsrechts. Hauptgrund für die höhere Regelungsdichte des Verfahrensrechts liegt in seiner Funktion, das Verhältnis Individuum-Verwaltung zu stabilisieren und die Verfahrensfairness sicherzustellen. Während das materielle Verwaltungsrecht vielfach offene inhaltliche Zielvorgaben macht und damit eine programmatische Stoßrichtung vorgibt, werden vom Verfahrensrecht eindeutige „Spielregeln“ erwartet. Das Verfahrensrecht soll der Entscheidungsbehörde wie auch den am Verfahren beteiligten Privaten klar abgesteckte Wege der Kommunikation und Entscheidungsfindung aufzeigen ${ }^{123}$. Es erscheint daher richtig, wenn etwa Fristen oder gewisse Formerfordernisse an die Kommunikation möglichst präzise normiert werden. Dies bedeutet indes nicht, dass im Verfahrensrecht jeglicher Ermessensspielraum der Behörde ausgemerzt werden

118 Kraft, in: ReNEUAL-Musterentwurf für ein EU-Verwaltungsverfahrensrecht. Tagungsband (FN 62), S. 154 (156 f.).

119 Schneider, in: ReNEUAL-Musterentwurf für ein EU-Verwaltungsverfahrensrecht. Tagungsband (FN 62), Einführung und Ergebnisse S. 7 f.

120 Fehling, in: ReNEUAL-Musterentwurf für ein EU-Verwaltungsverfahrensrecht. Tagungsband (FN 62), S. 143 (145).

121 Müller/Uhlmann, Rechtsetzungslehre (FN 112), Rn. 251.

122 Kraft, in: ReNEUAL-Musterentwurf für ein EU-Verwaltungsverfahrensrecht. Tagungsband (FN 62), S. 156.

123 Vgl. Kraft, in: ReNEUAL-Musterentwurf für ein EU-Verwaltungsverfahrensrecht. Tagungsband (FN 62), S. 156. 
sollte. Ein gewisses „Verfahrensermessen“ ist sogar unabdingbar, wenn das Verfahren mit Blick auf die konkreten Bedürfnisse im Einzelfall (z. B. Komplexität der sich stellenden Fragen, Anzahl Verfahrensbeteiligte, Dringlichkeit) im Sinne der Verfahrensökonomie effizient und effektiv geführt werden soll $^{124}$.

Zwei Beispiele aus dem Buch III sollen aufzeigen, dass die richtige Balance zwischen Bestimmtheit und Offenheit möglicherweise noch nicht durchgängig gefunden wurde:

Art. III-3 regelt die Grundsätze des fairen Verfahrens und der Unparteilichkeit. Nach Abs. 2 sind ,Verantwortliche Bedienstete im Sinne des Art. III-7 [...] verpflichtet, jedes finanzielle oder familiäre Interesse an einer Entscheidung ihren Vorgesetzten mitzuteilen, und an dieser Entscheidung nicht mitzuwirken. "Aus Sicht der am Verfahren direkt Beteiligten erscheint diese Bestimmung zu offen: Was sind ,finanzielle und familiäre Interessen “? ${ }^{125}$ Reicht das Eigentum an einer einzelnen Aktie eines am Verfahren beteiligten Unternehmens oder ein entfernte Verwandtschaft um einen automatischen Ausschluss zu bewirken? Betrachtet man die entsprechenden Vorschriften im deutschen, österreichischen oder Schweizer Recht, herrscht schnell Klarheit, zumindest, wenn es um familiäre Interessenkonflikte geht, denn diese werden minutiös umschrieben ${ }^{126}$. Eine solcherart bestimmte Regelung scheint gerade in Fällen des automatischen Ausschlusses vom Verfahren aber notwendig. Anders ist dies bei der Auffangnorm in Abs. 3: Alle anderen Interessenkonflikte führen nämlich zu einer individuellen Prüfung durch den Vorgesetzten, welcher dann entscheidet, ob ein Ausschluss von der Entscheidfindung angezeigt ist. Umgekehrt erscheint Art. III-3 in verwaltungsorganisatorischer Perspektive unnötig eng formuliert. Die Norm ist auf eine hierarchisch strukturierte Verwaltung mit monokratischen Entscheidträgern zugeschnitten. Was gilt nun aber, wenn die Verwaltungsbehörde kollegial organisiert ist? Oder wenn eine Person vorbereitend an einer Entscheidung mitwirkt, ohne gegen Außen Entscheidungsverantwortung zu tragen? Der Entwurfstext gibt hierauf keine Antworten ${ }^{127}$. Mit Blick auf einen möglichst weiten Anwendungsbereich und die Vielgestaltigkeit der Organe, Einrichtungen, Ämter und Agenturen der EU wäre eine in organisationsrechtlicher Hinsicht offenere Formulierung wünschenswert ${ }^{128}$.

Art. III-23 Abs. 5 regelt, wie dem Anspruch auf rechtliches Gehör im Verfahren zum Durchbruch verholfen werden soll. Ablauf und Gegenstand der

124 Vgl. Pünder, in: Allgemeines Verwaltungsrecht, (FN 38), S. 417 Rn. 13; S. 422 f. Rn. 19 f.; Schmidt-Aßmann, in: Grundlagen des Verwaltungsrechts II (FN 71), Rn. 104.

125 Kritisch auch Fehling, in: ReNEUAL-Musterentwurf für ein EU-Verwaltungsverfahrensrecht. Tagungsband (FN 62), S. 147.

126 Art. 10 Abs. 1 Bst. b und b ${ }^{\text {bis }}$ VwVG; $§ 20$ Abs. 5 VwVfG; $§ 36 a$ AVG; Art. 6 Abs. 1 Bst. $b$ und c LVG.

127 Vgl. immerhin die Erwägungen in den Erläuterungen: Schneider/Hofmann/Ziller (Hrsg.), ReNEUAL - Musterentwurf (FN 2), S. 72 Rn. 20.

128 Vgl. etwa Art. 10 Abs. 2 VwVG; § 7 Abs. 1 AVG; Art. 12 LVG. 
Anhörung wird dabei weitgehend dem Ermessen der Behörde überlassen. Dies gilt insbesondere für die Frage, ob eine Anhörung mündlich oder schriftlich erfolgen soll und ob Kreuzverhöre durchgeführt werden. Dieses Verfahrensermessen ist zu begrüßen, da es den Behörden erlaubt, den unterschiedlichen Verfahrenskulturen im europäischen Raum angemessen Rechnung zu tragen. Das Bedürfnis nach einem Kreuzverhör dürfte bei einer aus der Republik Irland stammenden Verfahrenspartei anders gelagert sein als wenn die Beteiligten aus Deutschland oder Frankreich stammen. Fragen wirft die Bestimmung aber in dem Punkt auf, wo sie der Behörde Handlungsanweisungen für die Ermessensausübung erteilt. Demnach „,berücksichtigt die Behörde [bei der Ermessensausübung] die gesetzlichen Ziele, die gesetzlichen Vorgaben, das Gewicht der Interessen des Anzuhörenden, die Bedeutung besonderer Verfahrensgarantien für die Interessen des Anzuhörenden sowie die Kosten der Gewährung solcher besonderer Verfahrensgarantien“. Die Bestimmung wiederholt vieles, was eigentlich selbstverständlich sein sollte (Berücksichtigung der gesetzlichen Vorgaben), sich bereits aus anderen Normen ergibt (Gewährung besonderer Verfahrensgarantien) oder schließlich gerade das Kernanliegen des Verfahrensermessens ist (Verfahrensökonomie). Der Entwurf erinnert hier eher an ein Handbuch der „Best Practice“. Möglicherweise sind solche Handreichungen eher kontraproduktiv, indem sie von den Entscheidträgern als Bevormundung empfunden werden, wodurch ihre intrinsische Motivation für die sorgfältige Wahrnehmung der eigenen Vollzugsverantwortung beeinträchtigt werden könnte.

Betrachtet man den Vorschlag des Europäischen Parlaments für ein EUVerwaltungsverfahren, so ist gegenüber dem ReNEUAL-Musterentwurf eine erfreuliche Straffung festzustellen. Der Charakter eines Handbuchs ist weitgehend verschwunden. Bezüglich Präzision besteht hingegen weiterhin Verbesserungspotential ${ }^{129}$. Schließlich wirft der Vorschlag des Parlaments neue Fragen auf, etwa bezüglich des nicht restlos geklärten Anwendungsbereichs und indem der „Verwaltungsakt“ (,administrative act“) als neuer Schlüsselbegriff eingeführt wird, allerdings ohne dafür eine aussagekräftige Definition zu liefern ${ }^{130}$.

\section{Schlussbemerkung}

Der ReNEUAL-Musterentwurf basiert auf einem einmaligen und fundierten Rechtsvergleich der EU-Mitgliedstaaten und reflektiert in gewisser Hinsicht ein europäisches ius commune proceduralis ${ }^{131}$. Er löst sich dabei aber von einem traditionellen, eng verstandenen Verfahrensverständnis und skizziert eine Reformagenda, welche die exekutive Informationsbeschaffung, In-

129 Vgl. etwa Art. 13 betreffend Interessenkonflikte, der sich stark an Art. III-3 des ReNEUAL-Musterentwurfs orientiert.

130 Schneider, in: ReNEUAL-Musterentwurf für ein EU-Verwaltungsverfahrensrecht. Tagungsband (FN 62), S. $134 \mathrm{f}$.

131 Pünder, in: Allgemeines Verwaltungsrecht (FN 38), S. 432 Rn. 30. 
formationsverarbeitung, Kommunikation, Entscheidfindung und Zusammenarbeit im europäischen Verwaltungsverbund breit erfassen will. Es ist eine Reformagenda, welche nicht nur für die Organe, Einrichtungen, Ämter und Agenturen der EU von Bedeutung ist, sondern darüber hinaus Ausstrahlungswirkung auf die Mitgliedstaaten und "Zugewandte Orte“ wie die Schweiz entfalten kann. Das Europäische Parlament hat sich im Juni 2016 in einem vorsichtigen ersten Schritt weitgehend auf eine schlanke kodifikatorische „Nachführung“ des individuellen Entscheidungsverfahrens beschränkt. Aus Sicht der Wissenschaft mag dies enttäuschend sein. Dass sich das Europäische Parlament von der Vorstellung einer bahnbrechenden Großkodifikation gelöst hat, könnte aber auch eine Chance darstellen, die im Musterentwurf dargelegten Reformen im Sinne eines Baukastensystems Schritt für Schritt voranzutreiben. Dieses modulartige Vorgehen würde auch eine stärkere Differenzierung bezüglich Innovationsgehalt, Regelungszielen und Regelungssprache erlauben. Aufgegriffen werden könnten dann auch andere Reformpostulate, welche im Musterentwurf nicht weiter vertieft wurden, etwa die Vereinheitlichung des verwaltungsinternen Rechtsschutzes oder Möglichkeiten alternativer Streitbeilegung. Gerade auch vor dem schweizerischen Erfahrungshorizont erscheint dieser pragmatische Ansatz auf lange Sicht vielversprechend. Schließlich macht dieser erste, noch eher zögerliche Schritt des Europäischen Parlaments deutlich, dass alles gesetzte Recht nur „vorläufiger Entwurf“ ist und „Recht und Rechtsstaat als Gegenstand ständig aufgegebener Bewältigung“132 zu verstehen sind. Weitere Schritte auf dem nun eingeschlagenen Weg sind zu tun.

\section{Abstract \\ ReNEUAL Model Rules on EU Administrative Procedure - a Swiss Perspective}

The article analyses the possible impact of the ReNEUAL Model Rules on EU administrative procedure for Switzerland, as well as the benefits of an additional Swiss perspective in the European discussion. Switzerland is not a member state of the European Union. Yet Switzerland is so closely connected to the European Union that codification of administrative procedure would sooner or later have spill-over effects on the Swiss legal system. Conversely, Swiss public administration with its multi-jurisdictional nature and pluralisation of actors is perfectly similar to that of the European Union in many respects. Experiences gained by Switzerland in connection with the codification of administrative procedure may therefore also be of value for the project in the European Union.

The authors of the ReNEUAL Model Rules deliberately drew them up as an innovative draft. In particular, this innovation manifests itself in a very broad

132 Bäumlin, Staat, Recht und Geschichte, 1961, S. 26, 46. 
approach which aims to comprehensively regulate as full a range of administrative activities as possible. What is especially noteworthy is the first-time attempt to regulate mutual assistance and administrative information management in a uniform and consistent manner. However, the European Parliament did not follow this comprehensive approach in June 2016 and instead suggested a modest "re-statement" of single decision-making. This pragmatic approach is strikingly similar to the concept on which the Swiss Federal Administrative Procedure Act (APA) of 1968 was based. This course of action may appear fainthearted, but from a Swiss perspective, it proved its worth step by step, since later reform steps have not been precluded. This is revealed, for instance, by the provisions on alternative dispute resolution added to the APA at a later stage. The procedural rules for Administrative Rule Making are strikingly similar to the proposals in Book II of the ReNEUAL Model Rules. They are, however not to be found in the Swiss APA, but in an act which regulates Administrative Rule Making together with the preliminary proceedings of legislation. A comparison with the Swiss legal system, therefore underlines that regulation of EU administrative procedure could be placed on an even broader basis than that proposed in the ReNEUAL Model Rules. Procedural laws in Switzerland regularly also contain provisions concerning administrative and judicial review. Bearing in mind the great fragmentation of the legal situation in the EU, a harmonisation of constitutional standards may well be useful in this respect. Besides some content-related aspects, this paper includes some critical references to the language and structure of the ReNEUAL Model Rules, which the author considers to be in need of improvement.

Even though the European Parliament is pursuing a rather modest approach for the time being, the ReNEUAL Model Rules will not forego their position as a reform agenda and point out what fields of activity of public administration should be codified for the reinforcement of democracy and the constitutional state in the medium-to-long term. However, the Model Rules not only outline a reform agenda for the EU's procedural law, but are also an expression of a European ius commune proceduralis. They can therefore serve as a model for the member states and associated countries such as Switzerland. 